\title{
Development of New Photogrammetric Software for High Quality Geo-Products and Its Performance Assessment
}

\author{
Jaehoon Jeong, Taeyoon Lee, Sooahm Rhee, Hyeon Kim and Taejung Kim ${ }^{\dagger}$
}

Image Engineering Lab, Inha University

\begin{abstract}
In this paper, we introduce a newly developed photogrammetric software for automatic generation of high quality geo-products and its performance assessment carried out using various satellite images. Our newly developed software provides the latest techniques of an optimized sensor modelling, ortho-image generation and automated Digital Elevation Model (DEM) generation for diverse remote sensing images. In particular, images from dual- and multi-sensor images can be integrated for 3D mapping. This can be a novel innovation toward a wider applicability of remote sensing data, since 3D mapping has been limited within only single-sensor so far. We used Kompsat-2, Ikonos, QuickBird, Spot-5 high resolution satellite images to test an accuracy of 3D points and ortho-image generated by the software. Outputs were assessed by comparing reliable reference data. From various sensor combinations 3D mapping were implemented and their accuracy was evaluated using independent check points. Model accuracy of 1 2 pixels or better was achieved regardless of sensor combination type. The high resolution ortho-image results are consistent with the reference map on a scale of 1:5,000 after being rectified by the software and an accuracy of 1 2 pixels could be achieved through quantitative assessment. The developed software offers efficient critical geo-processing modules of various remote sensing images and it is expected that the software can be widely used to meet the demand on the high-quality geo products.
\end{abstract}

Key Words : Geo product, software development, satellite image, mapping, DEM, ortho-image

\section{Introduction}

Geometric processing of various remote sensing data has been treated as an active research topic in the spatial information industry. The scale of the spatial information industry is expected to consistently increase and this brings close attention to the effective utilization of various remote sensing data. In particular, recent satellite sensor systems provide very high resolution and therefore, it is possible to generate high-precision geo-products from the satellite images. Many studies have demonstrated over the years that from satellite images geo-referencing accuracy of 1 2 pixels or better can be achieved by state-of-the-art photogrammetric techniques (Fraser et al., 2002; Dial et al., 2003; Noguchi et al., 2004; Büyüksalih et al., 2005; Jeong and Kim, 2009; Tong et al., 2010). Further investigations described that sub-meter

\footnotetext{
Received November 15, 2011; Revised February 15, 2012, Revised March 28, 2012; Accepted April 1, 2012.

${ }^{\dagger}$ Corresponding Author: Taejung Kim (tezid@inha.ac.kr)
} 
accuracy is supported by ultra high resolution of new satellites such as Geoeye-1, WorldView-1(Eckert, 2009; Fraser and Ravanbakhs, 2009). Connectedly, to generate fine and reliable geo-products such as DEMs and ortho-images, high resolution satellite images have been employed with suggestion of new or improved methods.

However, many previous studies including the cases listed above were limited to the suggestion of algorithm and several experiments for verification. For the growth and development of the spatial information industry, it is necessary that the proven methods are fully and widely exploited and this can be facilitated through software development and commercialization. Photogrammetric software commercialization is able to lead to wide opportunities for the application of various remote sensing data and provides great advantages in terms of the timely update and cost-reduction. However, there are not many software specialized for photogrammetric processing of satellite images, in particular to meet domestic demand. As domestic products, several products were released (Park, 2004) but mostly they are focused on general image processing functions. They are not specialized in photogrammetric processing for handling high resolution satellite images automatically. The government has discussed ways to vitalize domestic products from 2008 and aimed to controls about 80 percent of domestic market share, but we still depend heavily on the imported products at a high cost. This is mainly due to the lack of domestic products which can be alternatives to the imported products and therefore, the development of reliable domestic software for photogrammetric processing using high resolution satellite image, in particular KOMPSAT-2, can greatly contribute to national spatial information industry.

In this research, we introduce our newly developed photogrammetric software, "3DSuite", handling various remote sensing data for the production of high quality geo-products. This contains new techniques of an optimized sensor modelling, orthorectification and automated DEM generation for diverse remote sensing images. In particular, our software provides geometric integrations between different types of satellite images for 3D point determination, which have not been provided from many existing software. This can be a novel innovation toward a wider applicability of remote sensing data. This software is based on proven techniques developed in-house and the quality of this software is every bit as good as that of high-priced equivalents.

We briefly introduce a framework and characteristics of the developed software and then describe core functions in detail. Several testing processes have been implemented using the software and the outputs are evaluated to verify the software performances. An accuracy of the geometric processing from various remote sensing data is provided and the applicability of the developed software is suggested.

\section{Software framework and characteristics}

A framework of the developed software is shown in Fig. 1. The software is composed of five modules and each module is organically connected to one another and produces meaningful results.

In "Image" module, functions to input various remote sensing data and their metadata files are carried out. This module supports the majority of high resolution satellite images commonly used and recent aerial images such as airborne digital sensor (ADS) and digital mapping camera (DMC). In "Model" module, initial sensor modelling of inputted images with only the metadata files and precise sensor modelling with ground control points (GCPs) are selectively implemented by definition of users. 


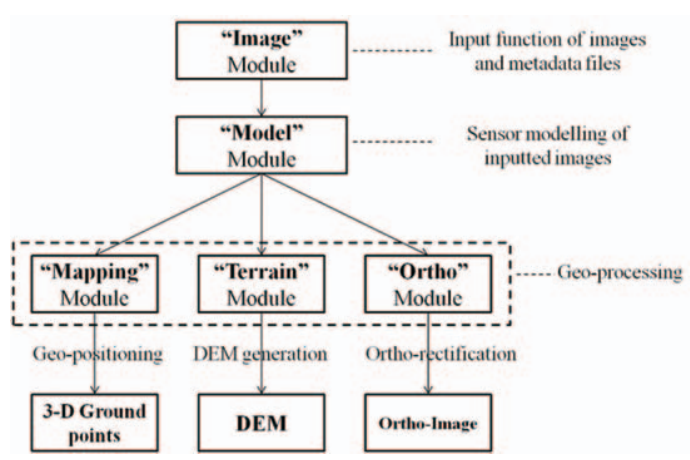

Fig. 1. Software framework.

This module provides rigorous sensor models (RSMs) and rational function models (RFMs) for satellite images modelling (Kim and Dowman, 2006; Grodecki and Dial, 2003) and functions of exterior orientation using collinearity equation for aerial images. After sensor modelling is precisely accomplished in "Model" module, desired geometric processing can be implemented in "Mapping", "Terrain" and "Ortho" modules. In "Mapping" module, new 3D ground points are generated by integrating the modelled images. In addition to conventional stereo model using single-sensor stereo images, the mapping module can handle geometric integrations between dual- and multi-sensor images for $3 \mathrm{D}$ point determination. This can be a novel innovation toward a wider applicability of remote sensing data, since 3D mapping of existing software have used images acquired by the same sensor so far. In "Terrain" module, fine spatial resolution DEMs are extracted through automatic image matching. As the matching algorithm, a newly developed correlation matching algorithm (3DCOM) that works in a 3D object space was employed. The matching algorithm was studied thoroughly through comparison with many existing matching algorithm and its performances were corroborated by automated DEM extraction from high resolution satellite images (Rhee and Kim, 2011). In "Ortho" module, orthoimages are generated through ortho-rectification process. This module includes functions of userdefinable resolution and coordinate system of output images and provides fine spatial resolution orthoimages that are comparable to a digital map on a scale of 1:5,000.

As mentioned above, the developed software offers the majority of the critical geo-processing modules of various remote sensing images. Table 1 shows sensor types and functionality supported by the software. From the Table, it can be observed that this software can be used for geometric processing using various high resolution remote sensing data. More detailed explanation regarding practical application will be discussed in the next section.

Table 1. Sensor types and functionality supported by the software

\begin{tabular}{c|c|c|c|c}
\hline \hline & Sensor modelling & 3D Mapping & DEM Generation & Ortho-rectification \\
\hline Kompsat-2 & RSM, RFM & 0 & 0 & 0 \\
\hline Spot-5 & RSM & 0 & 0 & 0 \\
\hline Ikonos & RFM & 0 & 0 & 0 \\
\hline QuickBird-1,-2 & RSM, RFM & 0 & 0 & 0 \\
\hline Geoeye-1 & RFM & 0 & 0 & 0 \\
\hline WorldView-1,-2 & RFM & 0 & 0 & 0 \\
\hline DMC & Collinearity Equation & 0 & 0 & 0 \\
\hline Ultracam & Collinearity Equation & 0 & 0 & 0 \\
\hline ADS & Collinearity Equation & 0 & 0 & 0 \\
\hline \hline
\end{tabular}




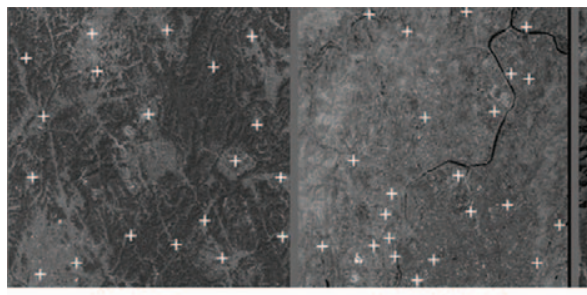

Spot -5

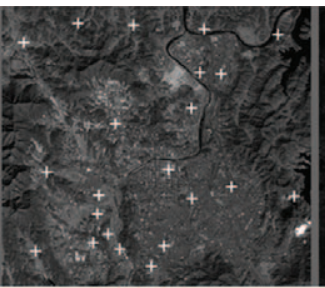

QuickBird

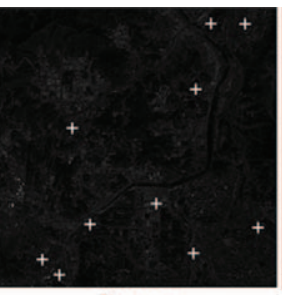

Ikonos

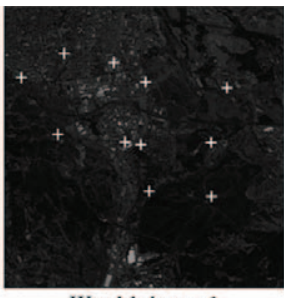

Worldview -1

Fig. 2. Satellite images used for experiments.
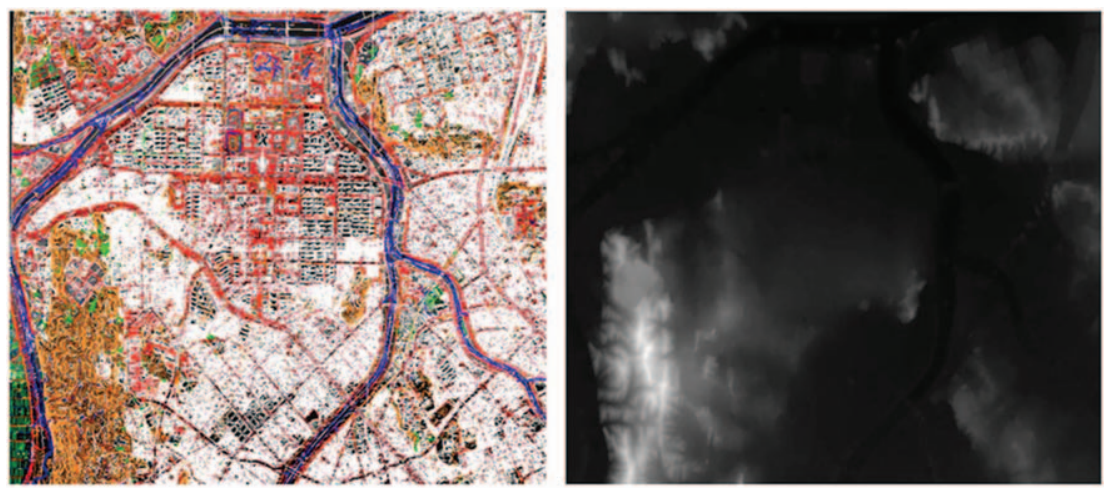

Fig. 3. Reference digital map (right) and extracted DEM (left).

Table 2. Properties of satellite images used

\begin{tabular}{c|c|c|c|c|c}
\hline \hline & Kompsat-2 & Ikonos & QuickBird & Spot-5 & Worldview-1 \\
\hline Acquisition Date & May 2007 & Feb 2002 & Jan 2005 & Dec 2003 & August 2008 \\
\hline Image resolution & $1 \mathrm{~m}$ & $1 \mathrm{~m}$ & $0.6 \mathrm{~m}$ & $2.5 \mathrm{~m}$ & $0.5 \mathrm{~m}$ \\
\hline Image size & $15000 \times 15000$ & $11004 \times 11004$ & $25044 \times 27552$ & $25000 \times 25000$ & $10000 \times 10000$ \\
\hline GCPs & 22 & 10 & 22 & 19 & 11 \\
\hline Upper Left Latitude & $36.43064712^{\circ}$ & $36.434715^{\circ}$ & $36.461548^{\circ}$ & $36.708210^{\circ}$ & $2.002915^{\circ}$ \\
\hline Upper Left Longitude & $127.2286807^{\circ}$ & $127.316632^{\circ}$ & $127.272200^{\circ}$ & $127.130920^{\circ}$ & $41.567226^{\circ}$ \\
\hline Lower Right Latitude & $36.32271078^{\circ}$ & $36.337224^{\circ}$ & $36.296970^{\circ}$ & $36.024062^{\circ}$ & $2.011705^{\circ}$ \\
\hline Lower Right Longitude & $127.4971326^{\circ}$ & $127.441324^{\circ}$ & $127.488637^{\circ}$ & $127.658122^{\circ}$ & $41.471734^{\circ}$ \\
\hline \hline
\end{tabular}

\section{Dataset and experiments}

In this section, our testing process to prove the performance of the newly developed photogrammetric software is described. Experiments for precise 3D mapping and ortho-rectification using various satellite images were carried out using the software. For tests Kompsat-2, Ikonos, QuickBird, Spot-5 high resolution satellite images covering the same area of Daejeon were mainly used. In addition, WorldView-1 image was used for DEM generation. Fig. 2 shows satellite images including GCPs distributions and their properties are presented in Table 2. A digital map on a scale of 1:5,000 was used to extract DEM for orthorectification and to assess ortho-image results. Fig. 3 shows the reference digital map and the extracted DEM.

We firstly inputted images and their metadata files to "Image" module. Using only metadata files, initial sensor modelling was established automatically by 
the module and the initial modelling errors were checked. We then established precise sensor modelling using GCPs for the input images. The modelling errors were checked visually and quantitatively. Sensor modelling accuracy is presented in the next section. As mentioned previously, "Model" module provides RSMs and RFMs for satellite images and both models were tested. In "Mapping" module, 3D ground object points can be generated from tie-points between the modelled images. We employed every possible combination from basic single-sensor stereo pairs such as Kompsat-2-Kompat-2 to multi-sensor combinations such as Kompsat-2-QuickBird-Spot-5. In "Terrain" and "Ortho" module, from the modelled images, fine spatial resolution DEMs and orthoimages were extracted respectively. The outputs are described in the next section.

\section{Results and performance assessment}

In this section, a performance of the developed software is assessed using output products by automatic geo processing. We are aiming for approximately $1 \sim 2$ pixel RMSE as 3D mapping accuracy and for by consideration of previous investigations (Büyüksalih et al., 2005; Kim et al., 2005; Oh et al., 2011) and for within the permissible accuracy of a map on a scale of 1:5000 notified by the National Geographic Information Institute as ortho-rectification accuracy.

We firstly established sensor models for test images and confirmed the modelling accuracy to verify geometric correction. In Table 3, we presented sensor model errors of initial modelling using only metadata files and precise modelling using GCPs. Sensor model accuracy shows that "Model" module furnishes reliable sensor model techniques for both RSMs and RFMs. This reliability can be demonstrated through subsequent experiments for 3D mapping, DEMs generation and ortho-rectification because their accuracy is directly involved in the accuracy of sensor models.

From the modelled images, geo-processing was implemented and the outputs were evaluated through comparison with reliable reference data. 3D mapping results were assessed using 14 independent check points, obtained through differential global positioning system (DGPS) processing. Table 4 shows an accuracy (root mean square error) of 3D mapping results. While there are a little differences due to spatial resolutions or stereo geometry of the integrated images, on the whole ground points were determined with an accuracy of $1 \sim 2 \mathrm{~m}$ in the horizontal direction and $1 \sim 3 \mathrm{~m}$ in the vertical direction, respectively. Assessment results represent that "Mapping" module provides a reliable 3D precise mapping service for even images from dualand multi-sensors, which is not supported in many

Table 3. Sensor modelling accuracy

\begin{tabular}{c|c|c|c|c|c}
\hline \hline \multirow{2}{*}{ Sensor } & \multirow{2}{*}{ Model } & \multicolumn{2}{|c|}{ Initial sensor model (pixel) } & \multicolumn{2}{|c}{ Precise sensor model (pixel) } \\
\cline { 3 - 6 } & & Column & Row & Column & Row \\
\hline \multirow{2}{*}{ Kompsat-2 } & RSM & 17.29 & 71.29 & 1.08 & 1.04 \\
\cline { 2 - 6 } & RFM & 16.34 & 69.97 & 0.96 & 1.17 \\
\hline \multirow{2}{*}{ Ikonos } & RFM & 4.05 & 9.34 & 0.76 & 1.29 \\
\hline \multirow{2}{*}{ QuickBird } & RSM & 9.89 & 26.13 & 1.28 & 1.42 \\
\cline { 2 - 6 } & RFM & 13.02 & 12.00 & 1.53 & 1.76 \\
\hline \hline
\end{tabular}


Table 4. 3D mapping accuracy

\begin{tabular}{|c|c|c|c|c|}
\hline Integration type & Image combination & Model & Horizontal Accuracy & Vertical Accuracy \\
\hline \multirow{8}{*}{$\begin{array}{l}\text { Single-sensor } \\
\text { model }\end{array}$} & \multirow{2}{*}{ Kompsat-2 stereo } & RSM & $1.86 \mathrm{~m}$ & $1.61 \mathrm{~m}$ \\
\hline & & RFM & $1.85 \mathrm{~m}$ & $2.09 \mathrm{~m}$ \\
\hline & \multirow{2}{*}{$\begin{array}{c}\text { Kompsat-2 multi-image } \\
\text { (three images) }\end{array}$} & RSM & $1.73 \mathrm{~m}$ & $1.50 \mathrm{~m}$ \\
\hline & & RFM & $1.81 \mathrm{~m}$ & $1.92 \mathrm{~m}$ \\
\hline & Ikonos stereo & RFM & $1.67 \mathrm{~m}$ & $0.74 \mathrm{~m}$ \\
\hline & \multirow{2}{*}{ QuickBird stereo } & RSM & $1.26 \mathrm{~m}$ & $0.86 \mathrm{~m}$ \\
\hline & & RFM & $1.27 \mathrm{~m}$ & $0.93 \mathrm{~m}$ \\
\hline & Spot-5 stereo & RSM & $2.78 \mathrm{~m}$ & $1.27 \mathrm{~m}$ \\
\hline \multirow{6}{*}{$\begin{array}{l}\text { Dual-sensor } \\
\text { model }\end{array}$} & Kompsat-2-Ikonos & RFM & $1.45 \mathrm{~m}$ & $2.74 \mathrm{~m}$ \\
\hline & \multirow{2}{*}{ Kompsat-2-QuickBird } & RSM & $1.78 \mathrm{~m}$ & $1.73 \mathrm{~m}$ \\
\hline & & RFM & $1.89 \mathrm{~m}$ & $1.58 \mathrm{~m}$ \\
\hline & Kompsat-2-Spot-5 & RSM & $2.07 \mathrm{~m}$ & $2.90 \mathrm{~m}$ \\
\hline & Ikonos-QuickBird & RFM & $1.52 \mathrm{~m}$ & $1.07 \mathrm{~m}$ \\
\hline & QuickBird-Spot-5 & RSM & $2.13 \mathrm{~m}$ & $3.37 \mathrm{~m}$ \\
\hline \multirow{2}{*}{$\begin{array}{l}\text { Multi-sensor } \\
\text { model }\end{array}$} & Kompsat-2-Ikonos-QuickBird & RFM & $1.46 \mathrm{~m}$ & $1.24 \mathrm{~m}$ \\
\hline & Kompsat-2-QuickBird-Spot-5 & RSM & $1.85 \mathrm{~m}$ & $2.15 \mathrm{~m}$ \\
\hline
\end{tabular}

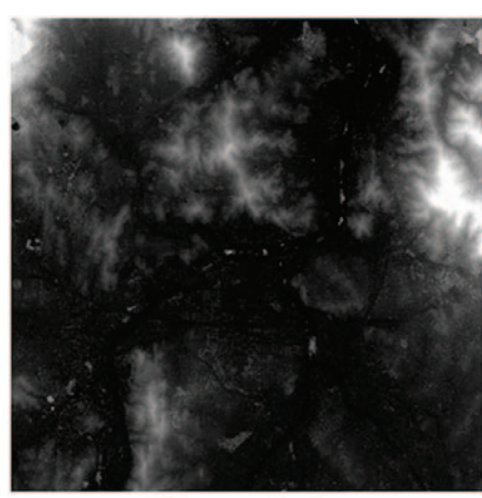

Kompsat-2

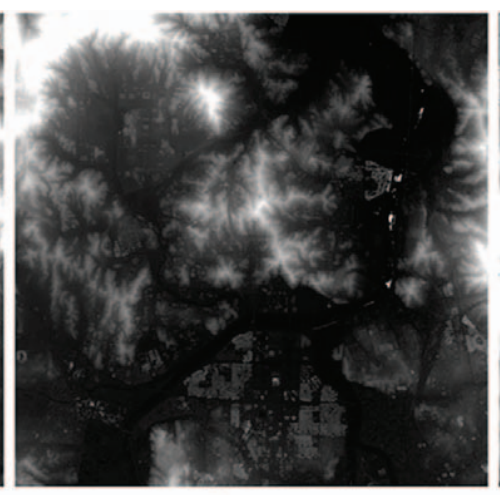

Ikonos

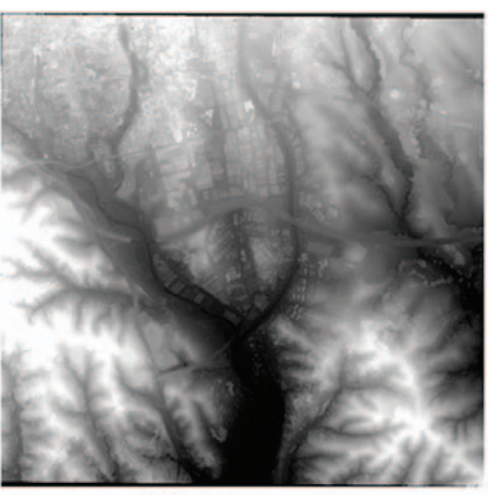

Worldview-1

Fig. 4. High resolution DEMs generated from the developed software (Rhee and Kim, 2011).

existing commercial software. We also confirmed earlier investigations presented an accuracy of 1 2 meter from Ikonos and Kompsat-2 and of 2 4 meter from Spot-5(Büyüksalih et al., 2005; Jeong and Kim, 2009; Vassilopoulou et al., 2004). We could conclude that our 3D mapping results are sufficiently good to produce very precise $3 \mathrm{D}$ metric information.

From "Terrain" module, fine resolution DEMs of 2 $\mathrm{m}$ spacing from Ikonos stereo and Worldview-1 stereo and of $5 \mathrm{~m}$ spacing from Kompsat-2 stereo were generated and they are presented in Fig. 4. It can be observed the DEMs describe very detailed surfaces. A study on the matching algorithm and DEMs accuracy assessment were already treated in our previous investigation (Rhee and Kim, 2011). These results support that automatic generation of reliable high resolution DEM using high resolution satellite images can be achieved by the "Terrain" module. In further research, the modules to generate DEM from aerial images will be updated and the matching algorithms to generate DEM from dualsensors stereo will be developed. 


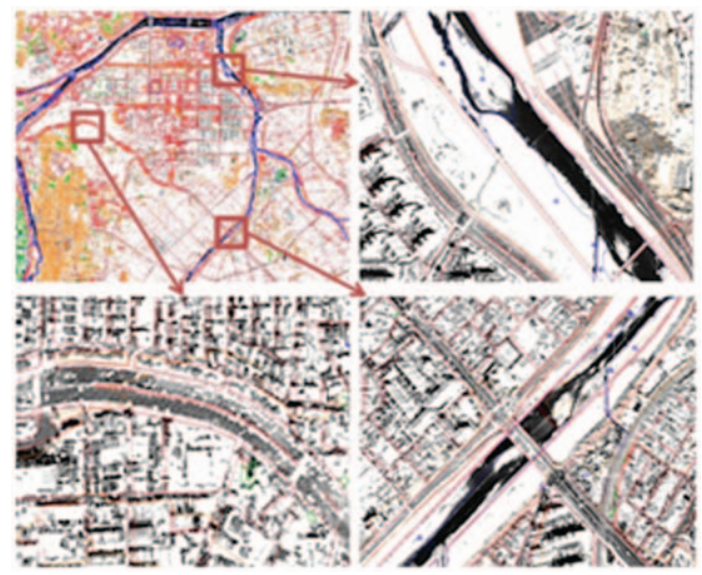

Fig. 5. Cross-sectional plots of overlap between reference map and Kompsat-2 ortho-image.

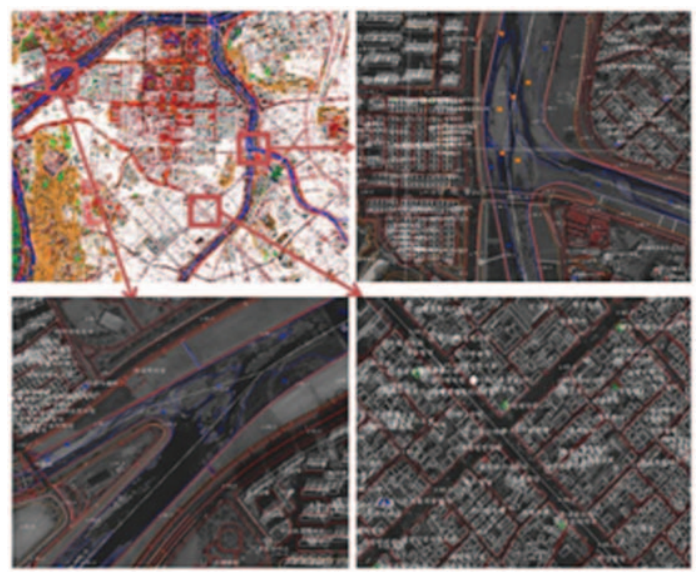

Fig. 7. Cross-sectional plots of overlap between reference map and Quickbird ortho-image.

From "Ortho" module, fine resolution Orthoimages of $1 \mathrm{~m}$ spacing from Kompsat-2, Ikonos and QuickBird images and of $2.5 \mathrm{~m}$ spacing from Spot-5 were extracted and a reference digital map on a scale of 1:5,000 was used for accuracy analysis. Fig. 5 8 show overlap images between the reference map and each ortho-image result and their cross-sectional plots. It is observed that the ortho-image results were consistent with the reference map after being orthorectified by the software. For quantitative assessment, we compared 10 points of the reference map with corresponding points of the ortho-images and calculated distance errors using RMS. Table 5 present

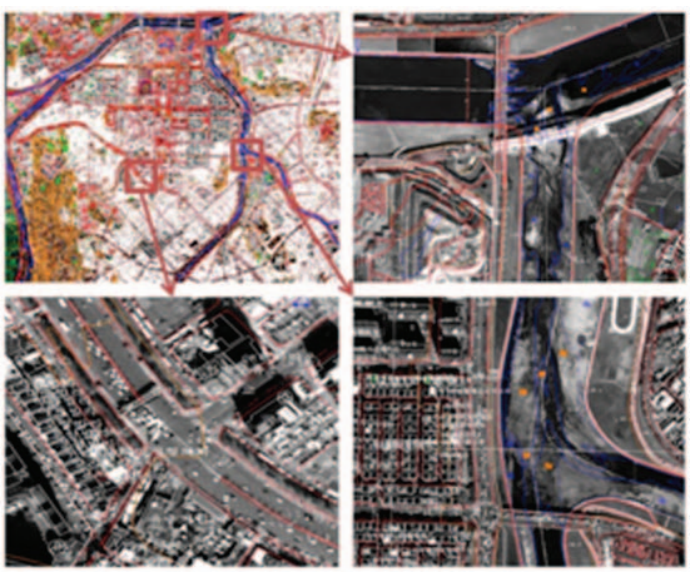

Fig. 6. Cross-sectional plots of overlap between reference map and lkonos ortho-image.

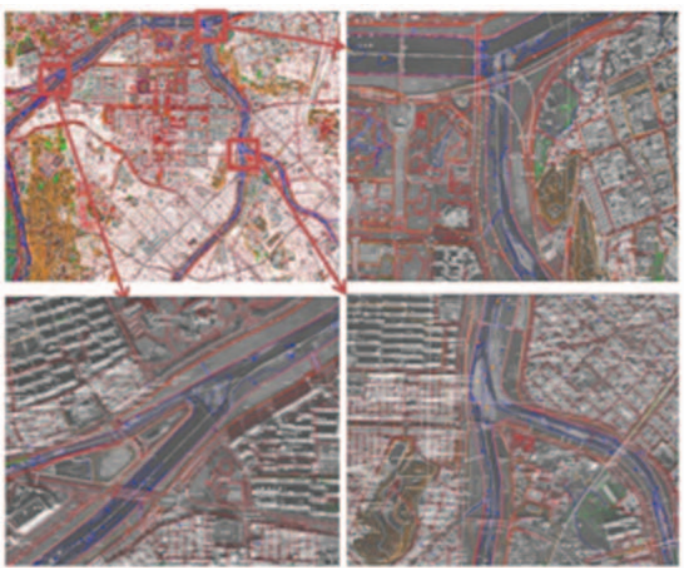

Fig. 8. Cross-sectional plots of overlap between reference map and Spot-5 ortho-image.

Table 5. Accuracy of Ortho-images generated by the developed software

\begin{tabular}{c|c|c|c}
\hline \hline & \multicolumn{3}{|c}{ RSM (meter) } \\
\cline { 2 - 4 } & X direction & Y direction & Distance \\
\hline Kompsat-2 & 2.37 & 1.61 & 2.86 \\
\hline Ikonos & 0.85 & 0.70 & 1.10 \\
\hline QuickBird & 1.15 & 1.47 & 1.87 \\
\hline Spot-5 & 1.89 & 3.16 & 3.68 \\
\hline \hline
\end{tabular}

the accuracy of ortho-images by the "Ortho" module. The overall results are comparable to previous investigation using QuickBird (Kang et al., 2008) and we also confirmed that it does not suffer by comparison with the accuracy available from existing 
software (Lee et al., 2009). We could observe that the accuracy is within the permissible accuracy of a map on a scale of 1:5000 notified by the National Geographic Information Institute and this facts imply our software can fully exploited for reliable digital map.

Through the performance assessments, we confirmed accurate 3D geo-positioning and high resolution DEMs and ortho-images. By using check points that are evenly distributed over various images, it could be observed that satisfactory results are achieved from software, regardless of data type or topographic characteristics. We could conclude that geo-processing results by our newly developed software show competent performances available for spatial information industry.

\section{Conclusion}

In this paper, our newly developed photogrammetric software has been introduced. To verify its performance, rigorous tests were carried out and the accuracy of the geometric processing using various high resolution satellite images was evaluated. 3D mapping results evaluated using independent check points showed an accuracy of 1 2 pixels or better regardless of sensor type combinations. For orthoimage results, it could be confirmed that they are consistent with the reference map on a scale of 1:5,000 and quantitative interpretation also provided satisfactory results. Overall, the assessment results are sufficient to demonstrate outstanding performances of the developed software. In addition to the functions suggested by this paper, the software provides most of the critical geo-processing modules of various remote sensing images and therefore it is expected that the software can be widely used to meet the demand on the high-quality geo products.

\section{Acknowledgment}

This work was supported by the National Research Foundation of Korea(NRF) grant funded by the Korea government(MEST)(2011-0009721) and by the supporting project to educate GIS experts.

\section{References}

Büyükssalih, G., G. Koçak, H. Topan, M. Oruç, and A. Marangoz, 2005. Spot revisited: accuracy assessment, dem generation and validation from stereo Spot-5 hrg images, The Photogrammetric Record, 20(110): 130-146.

Dial, G., H. Bowen, F. Gerlach, J. Grodecki, and R. Oleszczuk, 2003. Ikonos satellite, imagery, and products, Remote Sensing of Environment, 88(1/2): 23-36.

Eckert S., 2009. Validation of aircraft height extraction from Worldview-1 stereo data, International Journal of Remote Sensing, 30(22): 6053-6060.

Fraser, C.S., E. Baltsavias, and A. Gruen, 2002. Processing of Ikonos imagery for submetre 3D geopoisitioning and building extraction, ISPRS Journal of Photogrammetry \& Remote sensing, 56(3): 177-194.

Fraser, C.S. and M. Ravanbakhsh, 2009. Georeferencing accuracy of GeoEye-1 imagery, Photogrammetric Engineering \& Remote Sensing, 75(6): 634-640.

Grodecki, J. and G. Dial, 2003. Block adjustment of high-resolution satellite images described by rational polynomials, Photogrammetric Engineering \& Remote Sensing, 69(1): 59-68.

Jeong, J. and T. Kim, 2009. Comparison of orbitattitude model between Spot and Kompsat-2 
imagery, Korean Journal of Remote Sensing, 25(2): 133-143.

Kang, J.M., H.C. Yoon, J.K. Park, and Y.G. Kim, 2008. Application of QuickBird imagery for the production of digital map, Journal of the Korean Society of Surveying, Geodesy, Photogrammetry and Cartography, 26(1): 6371.

Kim, K., 2005. Computation of 3D coordinates from stereo images with RPCs, Korean Journal of Remote Sensing, 21(2): 135-143.

Kim, T. and I. Dowman, 2006. Comparison of two physical sensor models for satellite images: position-rotation model and orbit-attitude model, The Photogrammetric Record, 21(114): 110-123.

Lee, H.J., J.H. You, and Y.C. Koh, 2009. Analysis for practical use as KOMPSAT-2 imagery for product of geo-spatial information, Journal of the Korean Society for Geospatial Information System, 17(1): 21-35.

Noguchi, M. C.S. Fraser, T. Nakamura, T. Shimono, and S. Oki, 2004. Accuracy assessment of QuickBird stereo imagery, The Photogrammetric Record, 19(106): 128-137.

Oh, K.Y., H.S. Jung, W.J. Lee, and D.T. Lee, 2011. 3D Geopositioning accuracy assessment using KOMPSAT-2 RPC, Journal of the Korean Society of Surveying, Geodesy, Photogrammetry and Cartography, 29(1): 19.

Park, K., 2004. The Trend of Technical Development for high resolution satellite image processing, Korea Institute of Science and Technology Information, KOREA.

Sooahm, R. and T. Kim, 2011, DEM Generation and accuracy analysis from high resolution satellite images through a 3D correlation matching algorithm, Proc. of 2011 International Symposium on Remote Sensing, Yeosu, 2(4), 608-611.

Tong, X., S. Liu, and Q. Weng, 2010. Bias-corrected rational polynomial coefficients for high accuracy geo-positioning of QuickBird stereo imagery, ISPRS Journal of Photogrammetry \& Remote Sensing, 65(2): 218-226.

Vassilopoulou, S., L. Hurni, V. Dietrich, E. Baltsavias, M. Pateraki, E. Lagios, and I. Parcharidis, 2002. Orthophoto generation using Ikonos imagery and high-resolution DEM: a case study on volcanic hazard monitoring of Nisyros Island (Greece), ISPRS Journal of Photogrammetry \& Remote Sensing, 57(1/2): 24-38. 